\title{
Percutaneous Stone Surgery in Spina Bifida Patients-Are Stone-Free Rates Worth the Risk?
}

\author{
Rajeev Chaudhry, MD, ${ }^{1, *}$ Katherine M. Theisen, MD, ${ }^{2, *}$ Heidi A. Stephany, MD, ${ }^{1}$ Francis X. Schneck, MD, \\ Glenn M. Cannon, MD, and Michael C. Ost, MD ${ }^{1}$
}

\begin{abstract}
Purpose: Percutaneous stone surgery can be challenging in spina bifida (SB) patients due to their body habitus and spinal cord anomalies. A safe surgical approach may necessitate acceptance of lower stone-free (SF) rates. We seek to determine if anatomic complexity, as measured by spinal cord curvature and torso length, is associated with SF rates after percutaneous stone surgery in SB patients. We hypothesize that increasing anatomic complexity is associated with lower SF rates following percutaneous stone surgery.

Methods: We retrospectively reviewed all cases of percutaneous nephrolithotomy (PCNL) and percutaneous cystolitholapaxy (PC) in patients with SB at our institution. We calculated individual Cobb angles for scoliosis and kyphosis and measured torso lengths to serve as surrogates of anatomic complexity. These parameters were used to test for associations between anatomic complexity and SF rates.

Results: A total of 32 procedures were analyzed (15 PCNL, 17 PC). SF rates for PCNL and PC were $60 \%$ and $71 \%$, respectively. For both the PCNL and PC cohorts, there was no difference in age, preoperative stone burden, body mass index, operative time, or estimate blood loss between patients deemed SF or not stone free (NSF) after a single procedure. In the PCNL cohort, worsening scoliosis (higher Cobb angle) and lower SF rates trended toward statistical significance $\left(43^{\circ} v s .24^{\circ}, p=0.058\right)$. In the PC cohort, increasing torso length was associated with lower SF rates $(34.1$ vs. $28.5 \mathrm{~cm}, p=0.02)$.

Conclusions: Overall SF rates were modest in this population. Cobb angle measurements of scoliosis and kyphosis as well as torso length are novel tools for objectively defining anatomic complexity in the SB population and may help predict success following stone surgery.
\end{abstract}

Keywords: urolithiasis, Cobb angle, spina bifida, neurogenic bladder, torso length

\section{Introduction}

$\mathbf{P}$ ATIENTS WITH SPINA BIFIDA (SB) are at increased risk of both kidney and bladder stones and are more likely to undergo multiple stone procedures throughout their lifetime. ${ }^{1-4}$ Given a high prevalence of previous reconstructive surgeries (i.e., bladder augmentations, bladder neck closures/ reconstructions) and frequently large stone burdens, these patients often require percutaneous procedures such as percutaneous nephrolithotomy (PCNL) and percutaneous cystolitholapaxy (PC) for stone management. ${ }^{2}$

Surgical intervention in patients with SB is often complicated by other comorbidities frequently present in this population, including scoliosis, short stature, restrictive lung disease, obesity, and muscle spasticity. ${ }^{5}$ Specifically,
$44 \%$ to $90 \%$ of patients with SB have scoliosis and kyphosis. ${ }^{6-8}$ Spinal curvature, spinal hardware, and limb contractures all contribute to challenges with surgical positioning and access.

Nonetheless, several studies have demonstrated that PCNL is feasible in patients with SB. However, this approach can be humbling as complication rates range from $20 \%$ to $64 \%$ with modest stone-free (SF) rates of $9 \%$ to $60 \% .^{9-14}$

There are sparse data on outcomes following PC in this population, but abnormal skeletal anatomy can pose substantial challenges resulting in a technically difficult procedure. In addition, many of these patients have had previous abdominal surgeries (i.e., bladder augmentation and ventriculoperitoneal shunts), all of which can alter anatomic landmarks and increase the risk of complication.

\footnotetext{
${ }^{1}$ Division of Pediatric Urology, Children's Hospital of Pittsburgh of University of Pittsburgh Medical Center, Pittsburgh, Pennsylvania.

${ }^{2}$ Department of Urology, University of Pittsburgh Medical Center, Pittsburgh, Pennsylvania.

*These authors contributed equally (denotes cofirst authors).
} 
It seems logical that the anatomic complexity of SB patients can affect SF rates during percutaneous stone procedures, but there are little data to support this claim. Herein, we report our experience with percutaneous renal and bladder surgery for stone treatment in patients with SB. We hypothesize that increasing anatomic complexity defined as increasing scoliosis and kyphosis, and shorter torso lengths will be associated with lower SF rates following percutaneous stone surgery.

\section{Materials and Methods}

We performed a retrospective review of patients with SB who had undergone percutaneous stone procedures at our institution from January 2006 to June 2016. The data query identified patients with SB (ICD-9 741.00-741.03, 741.90741.93, or ICD-10 Q05.0-Q05.9) and neurogenic bladder (ICD-9 596.4-596.5 or ICD-10 N31.0, N31.9) who underwent PCNL (current procedural terminology [CPT] codes 50080, 50081) or PC (52317, 52318).

Factors analyzed in this study were determined a priori. Patient charts were reviewed for demographic information (age, gender, race, body mass index [BMI], and level of spinal defect), previous bladder surgery (bladder augmentation, bladder neck reconstruction, or urinary diversion), preoperative and postoperative stone burden, operative time, estimated blood loss, length of stay, and perioperative complications. Preoperative stone burden was determined based on preoperative imaging (CT or ultrasound). Postoperative stone burden was based on intraoperative fluoroscopy or postoperative imaging (CT or ultrasound).

Preoperative imaging was used to assign individual Cobb angles for measures of scoliosis and kyphosis. A Cobb angle is a measure of spinal curvature in degrees and is a standard measure for diagnosis, monitoring, and surgical planning of scoliosis/kyphosis in the orthopedic literature. It is the angle between two lines drawn perpendicular to the superior and inferior vertebral endplates of the curved segment ${ }^{15}$ (Fig. 1). A Cobb angle for the severity of scoliosis was measured using the largest curve in the thoracolumbar spine on anteroposterior or coronal imaging. Cobb angle for severity of kyphosis was measured using the largest curve in the lumbosacral spine on lateral/sagittal imaging. For the remainder of the article, we refer to scoliosis Cobb angle as "Cobb-S" and kyphosis Cobb angle as "Cobb-K." Finally, we measured torso length, which is the distance in centimeters between the thoracic inlet and a horizontal line drawn between a patient's iliac crests.

Patients were categorized based on the procedure completed: PCNL or PC. For each procedure group, analyses were performed between patients who were SF and those notstone free (NSF) after a single procedure. A small number of patients underwent both PCNL and PC procedures, but were only included in analysis if procedures were metachronous. Moreover, a single patient underwent two PCNL procedures, but they were performed on different sides on separate encounters. Procedures were not included in the analysis if they were repeat procedures for the same stone burden.

The primary outcome was to determine whether SF rates after PCNL or PC were associated with anatomical factors, including Cobb-S angle, Cobb-K angle, torso length, and BMI. Secondary outcomes included assessing whether SF rates were associated with operative time, estimated blood

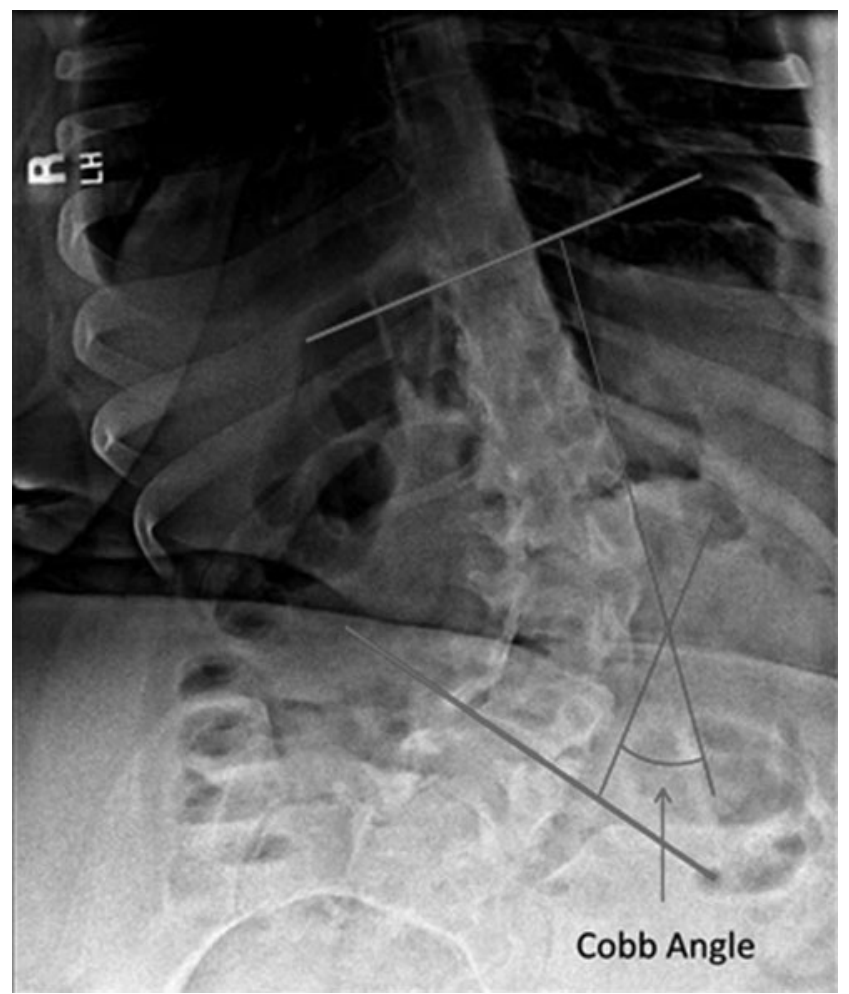

FIG. 1. Depiction of Cobb angle measurement for determination of the degree of scoliosis and kyphosis. The Cobb angle is the measurement between two lines drawn perpendicular to the superior and inferior vertebral endplates of the curved segment. ${ }^{15}$

loss, and perioperative complications following PCNL and $\mathrm{PC}$; the concern being that patients may suffer more harm if a surgeon's goal is to achieve SF status.

Data were analyzed using SPSS ${ }^{\circledR} 24$ statistical software. $\mathrm{SF}$ rates were calculated as percentages. Nonparametric variables were analyzed with the Mann-Whitney test in univariate analyses, using a $p$-value $<0.05$ to determine statistical significance. Multivariate analysis could not be performed reliably, given the size of cohort.

\section{Results}

Overall, 23 patients underwent a total of 32 procedures for our analysis. Median age at time of procedure was 23 years (interquartile range [IQR] 15-30, range 10-56 years), and our cohort was predominantly white, male, had lumbar spinal cord defects, and had a history of bladder augmentation with bladder neck procedure (Table 1). Of the 32 procedures, 15 were PCNL and 17 PC (Table 2).

In the PCNL cohort, 9 of 15 patients $(60 \%)$ were SF after a single procedure. There was no difference in age or preoperative stone size between SF and NSF groups. Median Cobb$\mathrm{S}$ angles trended toward being significantly higher in the NSF group than SF group at $43^{\circ}$ (IQR 38-63) vs. $24^{\circ}$ (IQR 13-39), respectively ( $p=0.058)$. The other anatomic factors of Cobb$\mathrm{K}$ angle, torso length, and BMI were not statistically different between the two groups. Operative time and estimate blood loss (EBL) were similar between the SF and NSF groups (Table 2). There were, however, more complications in the 
Table 1. Basic Demographics

\begin{tabular}{lc}
\hline Patients $(\mathrm{n}=23)$ & No. of patients $(\%)$ \\
\hline Age $(\mathrm{IQR})$, years & $23(15-30)^{\mathrm{a}}$ \\
Gender & \\
Male & $14(61)$ \\
Female & $9(39)$ \\
Race & \\
White & $21(91)$ \\
Black & $2(9)$ \\
Spinal cord lesion & \\
Lipomeningocele & $1(4)$ \\
Lumbosacral & $5(22)$ \\
Lumbar & $14(61)$ \\
Thoracic & $2(9)$ \\
Cervical & $1(4)$ \\
Bladder/bowel surgery & \\
Augmentation+bladder neck & $18(78)$ \\
Bladder neck only & $2(9)$ \\
Other urinary diversion & $1(4)$ \\
None & $2(9)$
\end{tabular}

${ }^{a}$ Median age with interquartile range.

$\mathrm{IQR}=$ interquartile range.

SF group; four complications $v s$. one complication in the NSF group. Complications included postoperative systemic inflammatory response syndrome (SIRS) in four patients and an intraoperative bleed in one patient (Table 3). Of note, the patient with an intraoperative renal bleed had a Cobb-S angle $>60^{\circ}$ (Fig. 2) and required a total of three procedures to achieve SF status.
Table 3. Postoperative Complications

\begin{tabular}{lcl}
\hline Complication & No. of patients & Procedure \\
\hline Postoperative SIRS & 4 & PCNL \\
Intraoperative bleed & 1 & PCNL \\
Urine extravasation & 1 & Cystolitholapaxy \\
Gross hematuria $^{\text {a }}$ & 2 & Cystolitholapaxy \\
Mental status change & 1 & Cystolitholapaxy \\
\hline
\end{tabular}

\footnotetext{
${ }^{a}$ Significant hematuria causing need for catheter replacement and irrigation of clots.

SIRS $=$ systemic inflammatory response syndrome.
}

In the PC cohort, 12 of 17 patients (71\%) were SF after a single procedure. There was a trend toward larger preoperative stone size in the NSF $v s$. SF group [6 cm (IQR 1.7-10) vs. $2.75 \mathrm{~cm}$ (IQR 2.2-4.1), respectively], but this was not statistically significant $(p=0.36)$ (Table 2$)$. There was no association between SF rates and anatomic parameters of Cobb-S angle, Cobb-K angle, or BMI. However, torso length was significantly longer in the NSF group compared with the SF group [34.1 cm (IQR 30.9-39.8) vs. $28.5 \mathrm{~cm}$ (IQR 27.1$30.1)$, respectively $(p=0.02)]$. Operative time and EBL did not differ between groups, and the number of complications was similar between groups with two in the SF group and two in the NSF group (Table 3).

\section{Discussion}

In our analysis of 32 percutaneous stone procedures in $\mathrm{SB}$ patients, our SF rates were modest at $60 \%$ and $71 \%$ for PCNL and PC, respectively. There are a few studies that evaluate SF

Table 2. Stone-Free Rates, Age, Stone Size, Body Mass Index, Cobb-S and Cobb-K Angles, and Torso Length Comparisons and Operative Factors Between Stone-Free and Not-Stone-Free Cohorts

\begin{tabular}{|c|c|c|c|}
\hline & Stone free & Not-stone free & $\mathrm{p}$ \\
\hline \multicolumn{4}{|l|}{ PCNL $(n=15)$} \\
\hline Stone burden after one procedure ${ }^{\mathrm{a}}(\%)$ & $9(60)$ & $6(40)$ & \\
\hline Age (IQR), years & $26(14-30)$ & $34(19-43)$ & 0.41 \\
\hline Preoperative stone size (IQR), cm & $2.6(1.5-2.9)$ & $3.1(1.8-3.8)$ & 0.38 \\
\hline BMI (IQR) & $25.6(20.7-28)$ & $30.3(26.9-37.8)$ & 0.19 \\
\hline Cobb-S angle (IQR), degrees & $24(13-39)$ & $43(38-63)$ & 0.058 \\
\hline Cobb-K angle (IQR), degrees & $46(17-57)$ & $69(61-71)$ & 0.12 \\
\hline Torso length (IQR), $\mathrm{cm}$ & $31(28.8-32.8)$ & $30(28.9-34.1)$ & 0.95 \\
\hline Operative time (IQR), minutes & $124(95.8-164.8)$ & $124(116.5-160.5)$ & 0.86 \\
\hline Length of stay (IQR), days & $4.4(3.5-4.6)$ & $2.1(1.9-2.6)$ & 0.02 \\
\hline $\mathrm{EBL}(\mathrm{IQR}), \mathrm{mL}$ & $40(17.5-50)$ & $20(10-175)$ & 1.0 \\
\hline No. of complications & 4 & 1 & \\
\hline \multicolumn{4}{|l|}{ Cystolitholapaxy $(n=17)$} \\
\hline Stone burden after one procedure ${ }^{a}$ & $12(71)$ & $5(29)$ & \\
\hline Age (IQR), years & $23(16.5-29.3)$ & $16(12-18)$ & 0.23 \\
\hline Preoperative stone size (IQR), $\mathrm{cm}$ & $2.8(2.2-4.3)$ & $6(1.7-10)$ & 0.36 \\
\hline BMI (IQR) & $27.1(19-31.1)$ & $22.5(18.3-28.6)$ & 0.87 \\
\hline Cobb-S angle (IQR), degrees & $15(12-44.5)$ & $8(8-13)$ & 0.29 \\
\hline Cobb-K angle (IQR), degrees & $61(54-72)$ & $74(66-101)$ & 0.28 \\
\hline Torso length (IQR), $\mathrm{cm}$ & $28.5(27.1-30.1)$ & $34.1(30.9-39.8)$ & 0.02 \\
\hline Operative time (IQR), minutes & $109.5(90.3-128)$ & $112(101-123)$ & 0.71 \\
\hline Length of stay (IQR), days & $1.4(1.2-1.9)$ & $0.4(0.4-1.7)$ & 0.56 \\
\hline EBL (IQR), mL & $10(10)$ & $10(5-10)$ & 0.43 \\
\hline No. of complications & 2 & 2 & \\
\hline
\end{tabular}

${ }^{\text {a }}$ Stone-free rates after first procedures with percentages in parentheses.

$\mathrm{EBL}=$ estimate blood loss; PCNL = percutaneous nephrolithotomy. 
FIG. 2. Scout films and CT scan of patient who sustained an intraoperative renal bleed during the access for PCNL. She had severe scoliosis with a Cobb angle $72^{\circ}$, making positioning, access, and manipulation of the kidney difficult. She required three procedures to be stone free. $\mathrm{PCNL}=$ percutaneous nephrolithotomy.

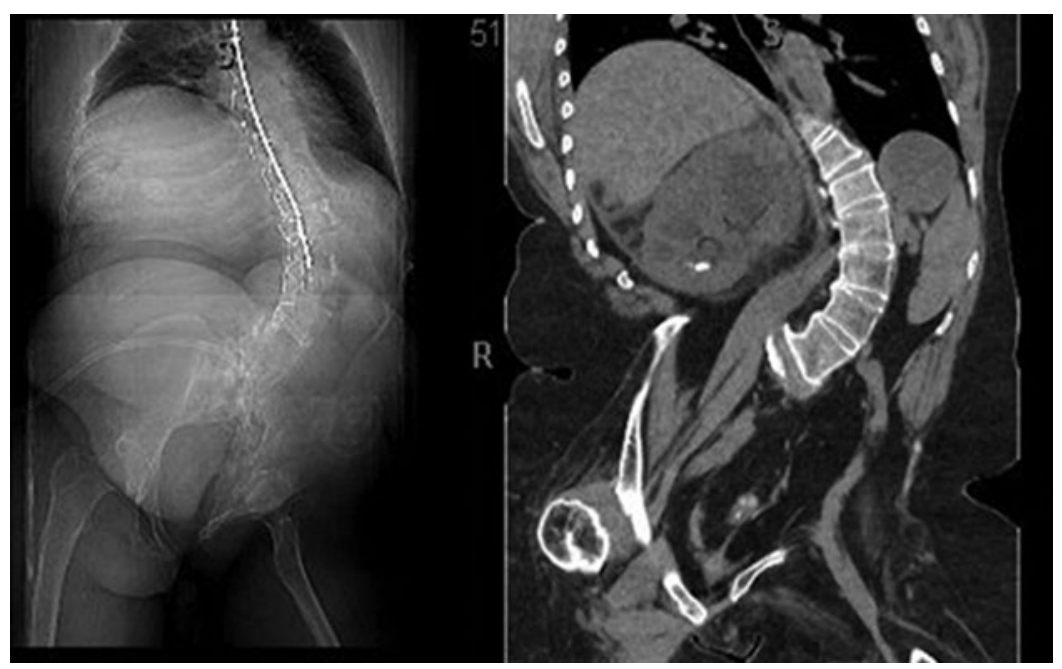

rates in patients with spinal anomalies, and the published rates are comparable to our findings, ranging from $49 \%$ to $60 \% .^{9-14}$ One could imagine that these modest SF rates compared to the general population could partly be explained by the anatomic complexity often encountered in patients with SB. Patients with SB are frequently nonambulatory and often have early-onset scoliosis and limb contractures. Furthermore, spinal hardware can result in a shortened trunk with spine and lung growth restriction, both of which can create challenges for surgical treatment of stone disease. The presence of hardware can also pose a challenge during fluoroscopy, as it can obscure view of the entire kidney (Fig. 3).

We hypothesized that lower SF rates would be related to increasing anatomic complexity, which we sought to describe in a novel manner. A Cobb angle is an objective measurement of spinal cord curvature that, to our knowledge, has not been discussed in urologic literature. This study is the first to look at this measurement, as well as torso length, to try and associate anatomic complexity with SF rates during percutaneous stone procedures.

In patients undergoing PCNL, the median Cobb-S angle was lower in the SF vs. NSF groups at $24^{\circ} v s .43^{\circ}(p=0.058)$, respectively. Although this result did not reach statistical significance due to our small sample size, we conclude that severe scoliosis can make percutaneous access and endoscopy of the kidney using a rigid nephroscope perilous, as was the case of our patient with a Cobb angle $>60^{\circ}$ (Fig. 2). We did not, however, find statistically significant associations between SF rates and Cobb-K angle, torso length, or BMI in the PCNL group.

For patients undergoing PC, we suspected that Cobb-K angle and torso length would more likely be associated with SF rates as severe kyphosis, and a short torso length could create a more challenging working space specific to this procedure. While there was no significant association between SF rates and Cobb-S angle, Cobb-K angle, or BMI in patients undergoing $\mathrm{PC}$, there was a significant association with SF rates and torso length, although opposite of what was anticipated. Patients with a shorter torso were more likely to be SF following a single PC. The authors suspect this result is a consequence of small sample size and represents a statically significant but not clinically significant finding.

In regard to our secondary outcomes, operative time and EBL were not associated with SF rates in either the PC or PCNL groups. Our overall complication rates were $33 \%$ for PCNL and $24 \%$ for PC. These rates are higher than seen in the
FIG. 3. Hardware can obstruct the view of the entire kidney, making the procedure difficult.

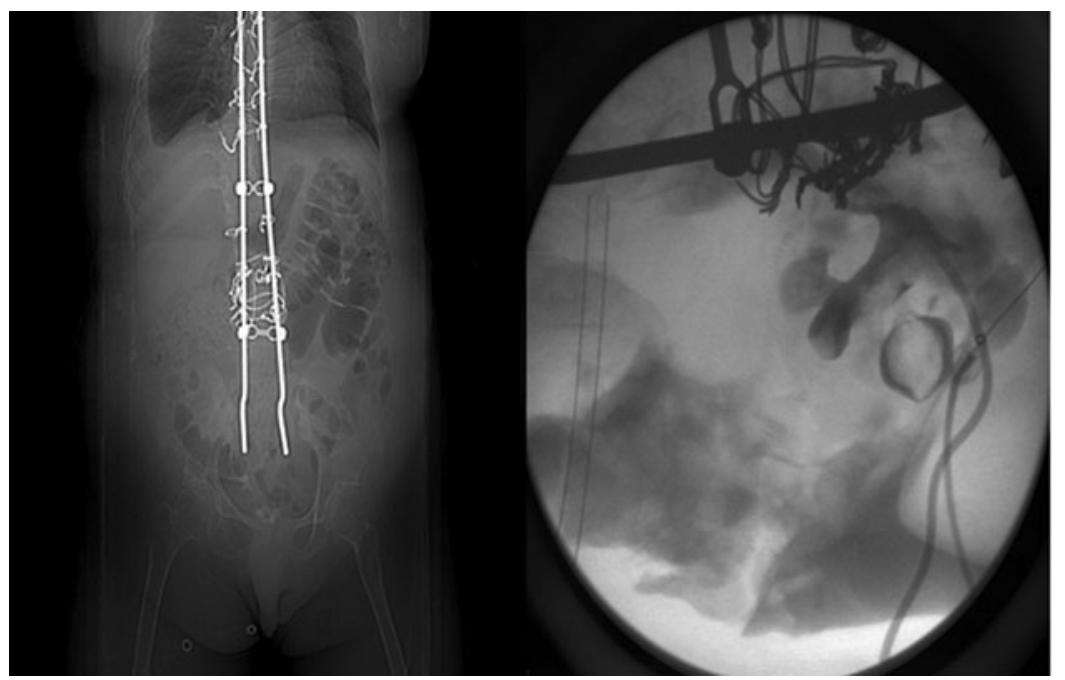


general population, but lower than previous studies of PCNL in patients with spinal deformities, which report total complication rates of $20 \%$ to $64 \%$, and major complication rates of $8.5 \%$ to $13.6 \% .^{11,13,16-18}$

Complications in our PCNL group were higher in the SF $v s$. NSF group (4 vs. 1, respectively). All four complications in the SF group were postoperative SIRS, despite our practice of rigorous utilization and treatment of preoperative urine cultures. However, this may not be unexpected, given SB patients typically form struvite or carbonate apatite stones. ${ }^{13,14}$ The rate of postoperative SIRS in our cohort $(27 \%)$ is similar to other studies of PCNL, which reported a rate of postoperative SIRS ranging from $30 \%$ to $64 \%$ in patients with spinal anomalies. ${ }^{13,16,18}$

In our PC group, the overall complication rate was modest at $24 \%$, with two complications in both the SF and NSF groups. The most serious complication was a patient who developed postoperative urinary extravasation from his augmented bladder after removal of stones totaling $>10 \mathrm{~cm}$ (Fig. 4). The initial percutaneous procedure was challenging due to his body habitus and stone burden and he ultimately required a secondary open cystolitholapaxy.

Given our finding that more patients in the PCNL group developed a complication after being rendered SF, we question whether the goal in this population should be achieving SF status in a single surgery. One patient developed a renal bleed during percutaneous access. This patient had severe scoliosis with Cobb-S angle $>60^{\circ}$ and her kidney was within the concavity of the scoliosis (Fig. 2). This made both access and subsequent stone removal difficult because of the drastic angles of her torso. This patient serves to highlight the anatomic

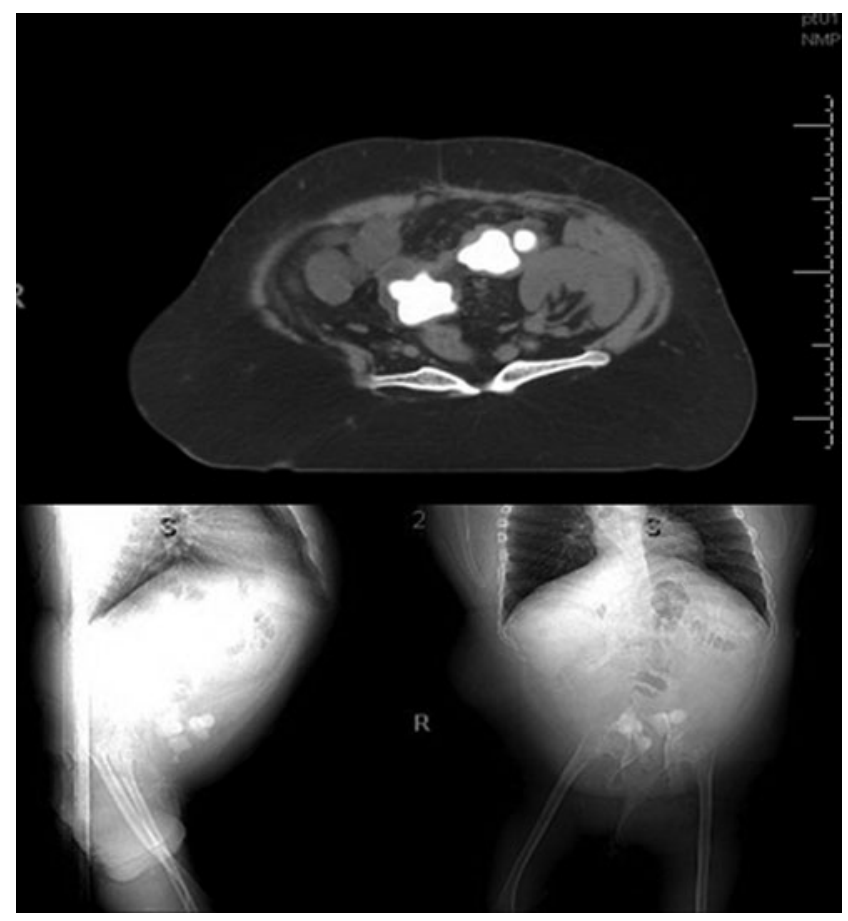

FIG. 4. CT scan and scout films for patient who developed urinary extravasation after percutaneous cystolitholapaxy. The axial images of the pelvis reveal stone burden $>10 \mathrm{~cm}$. This patient has barrel chest and short torso making access also difficult. complexity of this population and illustrates the propensity for higher complication rates. The patient ultimately required a total of three procedures to be rendered SF, but at what cost?

Overall, our results beg the questions-at what lengths should we pursue initial SF status in this patient population? Could use of Cobb angles and other objective measures of anatomic complexity help us predict which patients are less likely to achieve SF status in a single procedure and allow us to more readily accept lower SF rates in this population? There is a delicate balance between achieving maximal stone retrieval while avoiding morbidity in this often fragile and comorbid population. Due to the fragility of this patient population, we approach percutaneous stone surgery with a mentality similar to neurosurgeons-aim for the maximal safest resection. This is reflected in our results showing modest SF rates, but median operative times all approximately $\leq 2$ hours.

Limitations of this study are similar to those inherent to a retrospective study with a small sample size. Our sample size prohibited statistical significance, but certain trends were notable, specifically regarding higher Cobb-S angles and lower SF rates. No previous study has sought to objectively describe the degree of anatomic complexity in regard to scoliosis or kyphosis other than describing the kidney location in relation to the spinal cord concavity. The strength of our study lies in the attempt to overcome this limitation by using an objective measure of scoliosis and kyphosis used widely in the orthopedic literature to determine if anatomic complexity measured in this way was associated with outcomes of percutaneous stone surgery. Further larger scale studies will need to occur to determine the effect Cobb angles have on SF rates, difficulty of percutaneous access, and overall complication rates in patients undergoing stone surgery.

\section{Conclusion}

Percutaneous stone surgery in SB patients can be both a rewarding and humbling experience for urologists. Our experience shows modest SF rates after a single procedure. The severity of scoliosis and kyphosis objectively measured by Cobb angle may be predictive of a more challenging stone surgery, especially in regard to PCNL, and likely needs to be studied further. Percutaneous stone surgery has its benefits in this population, but understanding and having respect for anatomic complexity are important. Finally, perhaps rendering a patient SF should not be the focus or message to these patients, but rather, to attain a minimum residual stone burden in a single safe procedure within an acceptable operative time.

\section{Author Disclosure Statement}

No competing financial interests exist.

\section{References}

1. Ramachandra P, Palazzi KL, Holmes NM, Chiang G. Children with spinal abnormalities have an increased health burden from upper tract urolithiasis. Urology 2014;83: 1378-1382.

2. Schlomer BJ, Copp HL. Cumulative incidence of outcomes and urologic procedures after augmentation cystoplasty. J Pediatr Urol 2014;10:1043-1050. 
3. Palmer LS, Franco I, Kogan SJ, Reda E, Gill B, Levitt SB. Urolithiasis in children following augmentation cystoplasty. J Urol 1993;150(2 Pt 2):726-729.

4. Veenboer PW, Ruud Bosch JL, van Asbeck FW, de Kort LM. Urolithiasis in adult spina bifida patients: Study in 260 patients and discussion of the literature. Int Urol Nephrol 2013;45:695-702.

5. Werhagen L, Gabrielsson H, Westgren N, Borg K. Medical complication in adults with spina bifida. Clin Neurol Neurosurg 2013;115:1226-1229.

6. Piggott $\mathrm{H}$. The natural history of scoliosis in myelodysplasia. J Bone Joint Surg Br 1980;62-B:54-58.

7. Shurtleff DB, Goiney R, Gordon LH, Livermore N. Myelodysplasia: the natural history of kyphosis and scoliosis. A preliminary report. Dev Med Child Neurol Suppl 1976:126-133.

8. Samuelsson L, Eklof O. Scoliosis in myelomeningocele. Acta Orthop Scand 1988;59:122-127.

9. Goumas-Kartalas I, Montanari E. Percutaneous nephrolithotomy in patients with spinal deformities. J Endourol 2010;24:1081-1089.

10. Kara C, Resorlu B, Ozyuvali E, Unsal A. Is percutaneous nephrolithotomy suitable for patients with scoliosis: Singlecenter experience. Urology 2011;78:37-42.

11. Symons S, Biyani CS, Bhargava S, et al. Challenge of percutaneous nephrolithotomy in patients with spinal neuropathy. Int J Urol 2006;13:874-879.

12. Alsinnawi M, Torreggiani WC, Flynn R, McDermott TE, Grainger R, Thornhill JA. Percutaneous nephrolithotomy in adult patients with spina bifida, severe spinal deformity and large renal stones. Ir J Med Sci 2013;182:357-361.

13. Knox ML, Cantor AM, Bryant JE, Burns JR. Predictive factors for percutaneous nephrolithotomy outcomes in neurogenic bladder population. J Endourol 2012;26:823-827.

14. Nabbout P, Slobodov G, Mellis AM, Culkin DJ. Percutaneous nephrolithotomy in spinal cord neuropathy patients: A single institution experience. J Endourol 2012;26:1610-1613.

15. Kim H, Kim HS, Moon ES, et al. Scoliosis imaging: what radiologists should know. Radiographics 2010;30:1823-1842.

16. Culkin DJ, Wheeler JS, Nemchausky BA, Fruin RC, Canning JR. Percutaneous nephrolithotomy: Spinal cord injury vs. ambulatory patients. J Am Paraplegia Soc 1990; $13: 4-6$.

17. Culkin DJ, Wheeler JS, Jr., Nemchausky BA, Fruin RC, Canning JR. Percutaneous nephrolithotomy in the spinal cord injury population. J Urol 1986;136:1181-1183.

18. Rubenstein JN, Gonzalez CM, Blunt LW, Clemens JQ, Nadler RB. Safety and efficacy of percutaneous nephrolithotomy in patients with neurogenic bladder dysfunction. Urology 2004; 63:636-640.

Address correspondence to: Michael C. Ost, MD Division of Pediatric Urology Department of Urology Children's Hospital of Pittsburgh University of Pittsburgh Medical Center 4401 Penn Avenue 4th Floor Faculty Bldg. Pittsburgh, PA 15224

E-mail: michael.ost@chp.edu

$\begin{aligned} & \quad \text { Abbreviations Used } \\ & \mathrm{BMI}=\text { body mass index } \\ & \mathrm{CPT}=\text { current procedural terminology } \\ & \mathrm{CT}=\text { computed tomography } \\ & \mathrm{EBL}=\text { estimate blood loss } \\ & \mathrm{ICD}=\text { International Classification of Diseases } \\ & \mathrm{IQR}=\text { interquartile range } \\ & \mathrm{NSF}=\text { not-stone free } \\ & \mathrm{PC}=\text { percutaneous cystolitholapaxy } \\ & \mathrm{PCNL}=\text { percutaneous nephrolithotomy } \\ & \mathrm{SB}=\text { spina bifida } \\ & \mathrm{SF}=\text { stone free } \\ & \mathrm{SIRS}=\text { systemic inflammatory response syndrome }\end{aligned}$

